\title{
Los retos del gobierno multinivel y compartido para las políticas públicas. Del Estado relojero al Estado futbolista
}

André-Noël Roth Deubel 



\section{Los retos del gobierno multinivel y compartido para las políticas públicas. Del Estado relojero al Estado futbolista}

André-Noël Roth Deubel*

Recibido: Agosto 2006

Aceptado: Septiembre 2006
* Profesor investigador asociado. Departamento de Ciencia Política. Universidad Nacional de Colombia -sede Bogotá. anrothd@unal.edu.co

Polítólogo, Magíster y Doctor en Ciencias Políticas de la Université de Genève (Suiza). Ha sido profesor de los Departamentos de Ciencia Política de la Université de Genève (5 años) y de la Universidad del Cauca (10 años). Profesor invitado para cursos de postgrados o doctorados en las universidades del Valle, Externado de Colombia, ESAP, Antioquia, Universidad Nacional de Colombia, entre otras. Actualmente es Profesor investigador asociado en el área de las políticas públicas en la Universidad Nacional de Colombia y Profesor invitado en el 


\title{
LOS RETOS DEL GOBIERNO MULTINIVEL Y COMPARTIDO PARA LAS POLÍTICAS PÚBLICAS. DEL ESTADO RELOJERO AL ESTADO FUTBOLISTA
}

\author{
André-Noël Roth Deubel
}

\begin{abstract}
RESUMEN:
La acción administrativa está confrontada a una mutación profunda de la naturaleza de los problemas públicos que pretende abordar y, en lo posible, mitigar o resolver. Esta transformación de los problemas públicos hace necesario no solamente repensar las instituciones y administraciones públicas en función de fronteras y de segmentaciones nuevas (descentralización, federalismo, reforma administrativa, fusión, reparto de competencias, delegación, contratación, etc.) sino que implica una refundación profunda de la concepción misma de la acción pública. La necesaria reforma política administrativa implica también un cambio cultural. Para mostrar el sentido de estas mutaciones, es preciso partir de una presentación de la concepción de la administración tradicional de las políticas públicas para situar los cambios contemporáneos. Por medio del uso de diferentes metáforas profesionales se tratara de resaltar las líneas de fuerza que caracterizan la evolución de la labor administrativa para concluir con el esbozo de una propuesta de un Estado renovado que se adosa en una concepción democrática radical de la acción pública.
\end{abstract}

\section{PALABRAS CLAVE:}

Gobierno multinivel, políticas públicas, descentralización, reforma administrativa, Estado renovado

\section{ABSTRACT:}

The administrative action is confronted to a deep mutation of the nature of the public problems that tries to approach and, as far as possible, to mitigate or to solve. This transformation of the public problems not only makes it necessary to rethink the public institutions and administrations based on borders and in new segmentations (decentralization, federalism, administrative reform, fusion, distribution of competitions, delegations, hiring, etc.) but it also implies a deep reestablishment of the conception of the public function. The necessary administrative political reform implies as well a cultural change. In order to show the sense of these mutations, it is precise to start from a presentation of the conception of the traditional administration of the public policies to locate the contemporary changes. By means of the use of different professional metaphors the objective is to emphasizse the lines of force that characterize the evolutions of the administrative work to conclude with the outline of a proposal of a new renewed State that leans in a radical democratic conception of the public function.

\section{KEY WORDS:}

Government multilevel, public policies, decentralization, administrative reform, Renewed state 


\section{Desde el Estado relojero...}

Según la concepción tradicional la administración pública tiene como tarea la transformación de los problemas políticos en problemas técnicos proveyendo de manera racional los dispositivos organizativos y los medios necesarios a la resolución de dichos problemas en un territorio determinado. Para eso, el modelo administrativo más tradicional (moderno) consiste en la constitución de la administración en una organización de tipo burocrática idealtipificada por Max Weber. ${ }^{1}$ Tal organización se concibe entonces de manera vertical, con una línea de mando clara e uniforme, y con una segmentación tan vertical como horizontal, sintetizada en el organigrama, que garantiza la integración de las diversas actividades técnicas especializadas mediante una jerarquía funcional. El todo se sustenta y se legitima mediante el desarrollo de un derecho administrativo minucioso construido según el modelo de la famosa pirámide de Kelsen. Esta organización tiene la tarea de implementar en el territorio que le corresponde las políticas públicas decididas por la autoridad política a la cual está sometida. De modo que, en Colombia, el Estado nacional establece e implementa mediante su administración pública políticas válidas para lo que la jurisdicción considera como el territorio nacional, el Departamento hace lo suyo para el territorio departamental y el Municipio hace lo mismo en su jurisdicción. Del mismo modo que en los Estados federales, en los Estados descentralizados o unitarios la solución del buen funcionamiento del engranaje se busca en una repartición clara de las competencias respectivas de cada administración. Visto así, el problema de la administración de las políticas públicas se reduce entonces a un problema técnico de ajuste de la repartición de competencias de un lado y de capacidad técnica y financiera de los funcionarios del otro. Se trata entonces de yuxtaponer, tanto horizontal como verticalmente, diferentes segmentos o fragmentos de políticas públicas sectoriales y territoriales de manera que se complementen sin que interfieren entre si. Cada administración dispone de soluciones y de medios

Doctorado en Ciencias Sociales, Niñez y Juventud de la Universidad de ManizalesCINDE. Autor, entre otros, de "Políticas públicas. Formulación, implementación y evaluación", Ediciones Aurora, 2002 (5 ediciones), "Discurso sin compromiso. La política pública de los derechos humanos en Colombia, Ediciones Aurora, 2006. Director del grupo de investigación "Análisis de las políticas públicas y de la gestión pública" (Categoría B, 2006). Miembro de los grupos de investigación "Gestión y Políticas públicas" de la Universidad del Valle (Categoría A, 2006) y "Políticas y programas de Niñez y Juventud y Desarrollo Social" de la Universidad de ManizalesCINDE (Categoría B, 2006).

1 Weber, Max, Economía y Sociedad, México, FCE, 1987. Ver en particular pág. 716752. 
preconstruidas especializados que configuran su segmento de la política pública, desconociendo los otros segmentos. Según esta concepción de la administración, los problemas que pueden surgir de esta construcción son similares a las dificultades técnicas encontradas por un relojero en calibrar perfectamente las diferentes piezas de un complicado mecanismo para que todos los movimientos se efectúen de manera coordinada y precisa. Es el Estado relojero.

El mecanismo relojero es el modelo metafórico del Estado y la administración pública modernos. Es el modelo que operaba con la Constitución colombiana de 1886, basado en la sectorización y la desconcentración de algunas funciones en los niveles departamentales y municipales, en donde los gobernadores y alcaldes eran simples agentes administrativos considerados como engranajes del gobierno nacional. La lógica profunda del modelo consiste en lograr la resolución de un problema mediante la división de este en una multitud de "problemitas" susceptibles de ser resueltos por intervenciones administrativas especializadas, tal como un engranaje de un reloj. La sumatoria mecánica de estas intervenciones, aisladas e independientes las unas de las otras, es la que debía permitir la resolución del problema general.

Es de anotar que este modelo relojero corresponde a un particular punto de vista: es él de la racionalidad científica moderna. Pertenece a la creencia positivista de que existe una Razón independiente de los valores (value free), una razón objetiva que puede ser descubierta mediante el método científico positivo. Esta concepción de Razón objetiva se trasladó a la actividad del Estado. Es el fundamento ideológico del Estado moderno. Este debería actuar en base a una razón científica que trasciende los intereses particulares que se expresan en la sociedad. De modo que el Estado actúa de manera independiente, soberano, y con la legitimidad que le da el hecho de actuar en base a un conocimiento científico, exterior a toda pasión política o a alguna sumisión a intereses particulares, que correspondería automáticamente al bien público.

Sin embargo, a pesar de que esta construcción organizacional racional, porque pensada para la eficacia y la eficiencia sobre el modelo industrial y científico (positivista), parecería idónea, no logra resolver los problemas públicos tal como son realmente y como los vemos actualmente. Tal y como el modo tayloriano de producción masificada de bienes industriales tiende a dislocarse, el modo weberiano, conceptualmente similar, de administración de la producción de los bienes y de los valores públicos aparece en crisis. Este modelo burocrático resulta incapaz de responder a la diversidad y complejidad de los problemas, a las situaciones y necesidades político administrativas expresadas por los ciudadanos contemporáneos. Igualmente, la imagen de un Estado que actuaría de forma objetiva, científica y racional, ya no es creíble. De hecho, es 
toda la ideología de la modernidad, con la cual se consideraba que la acción del Estado estaba guiada por la objetividad de la ciencia y por la razón suprema del bien público, que ha perdido vigencia y credibilidad.

Como primera respuesta, se consideró necesaria la adopción de una nueva arquitectura institucional que distiende un tanto los contactos entre partes del reloj. En vez de una transmisión rígida, simbolizada por piezas mecánica directamente en contacto, se considero necesario introducir un juego entre estas piezas y acoplarlas mediante una correa más o menos templada: la descentralización. En Colombia, con el cambio de Constitución ocurrido en 1991, se ratifica (municipios desde 1986) y se introduce (departamentos) una descentralización en estos niveles (incluyendo también un cuarto nivel: el resguardo). De forma general, la descentralización implica entonces que la legitimidad y la autoridad políticas se fragmenten, se conceden márgenes de autonomía a los diferentes niveles institucionales. De la lógica política y administrativa unitaria se pasa a una lógica más plural, policéntrica. Los territorios municipales (y departamentales) ya no son una simple subdivisión administrativa del territorio nacional, sino que adquieren una cierta autonomía frente a éste. Ya no hay una administración nacional de las políticas públicas en un territorio, pensado como uniforme, organizada de arriba hacia abajo con una clara y única línea de mando encabezada por el Gran Relojero, sino que emerge una pluralidad de administraciones territoriales que disponen de una cierta autonomía política y administrativa.

Eso significa el paso de una organización complicada (mecánica) a un sistema más complejo de organizaciones que tienen que interrelacionarse para actuar en conjunto y coordinar sus políticas respectivas tanto horizontal como verticalmente, ya que se introdujo de esta forma un "juego" entre las diferentes piezas del reloj: cada elemento tiene cierta autonomía para encontrar su modalidad específica-en función de las diferentes sensibilidades locales-de encajar en el mecanismo relojero. Las instancias de planeación y el Plan generan directrices de orientación a los entes descentralizados con la finalidad de lograr de manera más flexible la integración de las políticas públicas desarrolladas. Sin embargo, la herramienta, a pesar de sus diferentes modalidades - de las más rígidas (unitarismo) a las más flexibles (federalismo)- tiende a ser similar en su concepción general. La diferencia fundamental reside en que la descentralización -el aflojo de la transmisiónpermite un mayor aguante ante las perturbaciones: mientras un solo grano de arena puede bloquear el reloj, en un mecanismo con más juego entre piezas, es posible que siga funcionando a pesar de la presencia de granos de arena. El mecanismo tendrá talvez unos "achaques" pero hasta cierto punto lograra seguir en funcionamiento.

Sin embargo, estas reformas no resuelven todo: los problemas de coordinación efectiva de las diferentes ac- 
tividades administrativas se agudizan debido al margen de autonomía que disponen los diferentes actores institucionales. De administrativa, la coordinación se vuelve más política. De hecho, surgen entonces una multitud de políticas públicas -nacionales, departamentales, municipales- yuxtapuestas que responden a autoridades políticas y administrativas no totalmente integradas ni completamente sometidas a un orden jerárquico claro e unívoco: traslapos, descoordinación, duplicación de esfuerzos son el denominador común de la nueva arquitectura institucional. El relojero, al introducir juego entre sus piezas, tiende a perder el control de su sofisticado aparato, que se parece más a una máquina a Tinguely ${ }^{2}$, un tanto incontrolable e imprevisible, o en el peor de los casos a un especie de Frankenstein que escapa al control de su creador, que a un reloj de alta precisión que da la hora exacta con seguridad. La descentralización (o el federalismo) puede considerarse entonces como una manera de "aceitar" o de "aflojar" el mecanismo. La administración pública, en particular debido al continuo crecimiento de las funciones, sigue en crisis y la sombra del universo kafkiano sigue rodando. Crisis que se debe tanto al crecimiento y expansión de la acción pública a prácticamente todos los ámbitos de la vida humana como al cambio tanto de la naturaleza de los problemas que pretende resolver como de las expectativas de los ciudadanos. Disten- der las correas no basta si, al mismo tiempo, no opera un cambio de cultura organizacional.

El paso de la ideología de la complicación (mecánica) a la ideología de la complejidad (actores autónomos que tienen que cooperar) no está aún inscrito en la cultura política administrativa dominante (a pesar de la retórica oficial de fachada). En muchos casos la cultura política administrativa tiende a quedar pegada a una visión mecánica de la descentralización y de la acción pública. Se intenta resolver problemas complejos con soluciones complicadas. De allí que se insiste en el problema de la distribución de las competencias, de la reglamentación jurídico administrativa detallada y de la autoridad jerárquica, es decir se sigue pensando y actuando según la metáfora relojera, es decir mecánica.

Eso significa, en la práctica, una imposibilidad o por lo menos una gran dificultad para la coordinación administrativa, en la medida en que los problemas actuales ya no se dejan resolver mediante simple yuxtaposición o segmentación de tareas administrativas, sino mediante soluciones complejas que implican la deliberación y la negociación políticas para la realización de actividades en conjunto y no solo en complemento. Es decir, se sustituye la administración de lo complicado por la gestión de la complejidad o, para retomar a Engels en su visión del mundo futuro ideal, ya no se trata de pasar del gobierno de los hombres

2 Artista suizo que construye esculturas móviles con material industrial. 
a la administración de las cosas, que expresa esta visión racionalista y cientista de la modernidad, sino de la administración de las cosas al autogobierno de los hombres. Es decir, de reconocer la complejidad de la vida y de lo humano, cuya gestión no puede reducirse a una administración mecánica de objetos (ideal de la ciencia positiva). La necesidad de la deliberación política se debe a que los problemas a los cuales las políticas públicas intentan responder desbordan ampliamente los límites territoriales geográficos sectoriales establecidos por las divisiones administrativas. En otras palabras es el fracaso de la ilusión cientista del siglo XIX y durante buena parte de siglo XX, que se expresó en el pensamiento taylorista.

Debemos pasar así, según mi definición, de la administración pública a la gestión, o mejor, a la autogestión pública. El cambio no es sólo semántico, sino que recubre entonces un cambio en la manera de relacionarse con el otro, de concebir y desarrollar la actividad administrativa. Ya no se trata de ordenar burocráticamente, es decir obtener la cooperación mediante la obligación, la autoridad de la norma, sino mediante procesos participativos y deliberativos, es decir actuar políticamente, para obtener una cooperación libre tanto intra- como extra- administrativa: se pasa así de la coordinación técnica (burocracia, autoridad jerárquica) -el paradigma mecánico-a la coordinación política (deliberación, negociación, acuerdo, contrato) -el paradigma complejo- como modelo dominante, aunque no necesariamente exclusivo, para la acción pública. La (auto)gestión pública consiste en encontrar las modalidades de acción que incluyen segmentos administrativos de diferentes niveles y sectores, organizaciones privadas y $\mathrm{ONG}$, etc., para lograr el objetivo en conjunto (y no en complemento).

Es en este sentido, que pretendo resignificar diversos conceptos que se han generalizado en los estudios y el lenguaje político administrativos contemporáneos. Considero que estos cambios en el vocabulario reflejan de una cierta forma lo que se entiende como el paso de la ideología "moderna" a la "postmoderna" que puede ser presentada con el Cuadro 1. Este cambio implica una caída del Estado de su piedestal "racional" que correspondió a una cierta manera (autoritaria) de gobernar el mundo y a su devenir: el ineluctable desencanto progresivo del mundo debido al avance de la racionalidad que Weber había percibido, o la progresiva administración de las cosas en vez del gobierno de los hombres. 
Cuadro 1

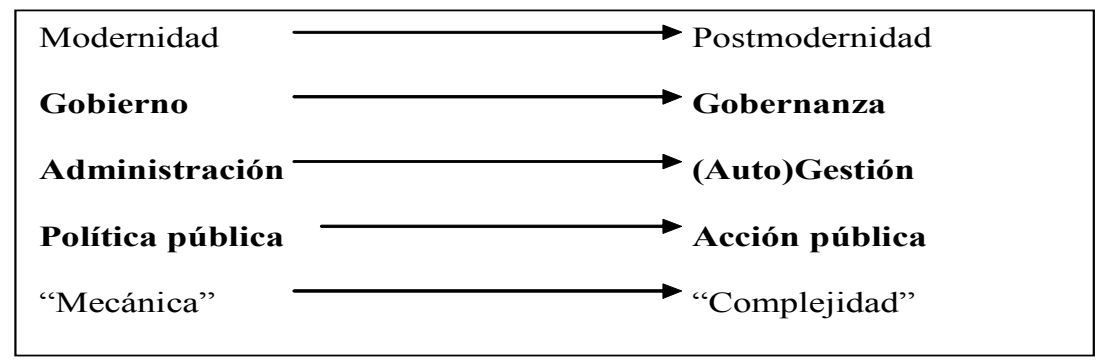

\section{Digresión}

Sin embargo, es necesario precisar que, en realidad, este cambio se debe más al cambio de paradigma dominante, es decir a la sustitución de nuestros lentes de observación y de comprensión de la realidad que nos hace ver de forma diferente el mundo, que a una progresiva complejificación de éste. De hecho, creo que, en los siglos XVIII y XIX, con el movimiento de la Ilustración $^{3}$, vehículo ideológico de esta particular visión del mundo y de la razón moderna, se asiste a un cam- bio en las relaciones de fuerzas entre Estado y grupos de interés ${ }^{4}$, -la burguesía ascendente haciendo pasar sus intereses como siendo basados en una razón externa (científica, positiva) a ella y, así mismo, logrando legitimar su control del Estado para permitir la defensa de estos intereses como siendo racionales y justos porque "objetivamente" comprobados por el método científico ${ }^{5}$. La Declaración de los Derechos del Hombre y del Ciudadano de 1789 será uno de estos instrumentos ideológicos para derrotar política y

3 El cuál no ha sido un movimiento homogéneo en su momento como aparece con la distancia del tiempo. Estudios históricos comparativos muestran por ejemplo que hay diferencias entre les Lumières francesas, el Enlightement anglosajón y el Aufklärung germánico, así como al interior mismo de estas agrupaciones lingüístico culturales, en particular en cuanto al lugar de la religión.

4 Ver Papadopoulos Y. "Gouvernance et transformations de l'action publique: quelques notes sur l'apport d'une perspective de sociologie historique", en Laborier P., Trom D., Historicités de l'action publique, PUF, 2003, pp. 119-135.

5 Es de anotar que el marxismo, y en particular en su interpretación ortodoxa posterior a Marx, caerá en la misma trampa, considerándose como más científico, más verdadero que la verdad burguesa. Y como sólo puede haber una sola verdad, se entiende sus tendencias totalitarias, el desprecio al pluralismo democrático y el trato a los "disidentes": la excomunicación y/o el campo de reeducación. Recordaré también que Lenin, y el marxismo en general, fue el más entusiasta con el progreso de la ciencia en general y, en particular, con la administración científica de F. Taylor. 
culturalmente a los intereses de los señores feudales y su tipo particular de relación con las autoridades políticas derivado, y legitimado, según ellos, a partir de una razón de esencia divina. Un análisis detallado del régimen feudal mostraría también la complejidad de las redes y de las lealtades cruzadas que prevalecían en el régimen político del medioevo. El "lente moderno" ha minimizado la complejidad de las interrelaciones e interdependencias entre actores para magnificar el Estado racional y considerar estas relaciones como siendo restos de premodernidad que serán arrasados con el "progreso"6. El siglo XIX, que fue teatro de una era de innovaciones técnicas importantes, permitió la constitución de una causa común, una alianza, entre ciencia y Estado a nombre del progreso alcanzado por medio de una nueva definición de la razón, considerada como científica.

De un cierto modo, la dificultad contemporánea de la administración pública en gobernar nuevos territorios de acción pública corresponde no sólo al cambio de la naturaleza de los problemas, sino también a un cambio en la percepción de los problemas (cambio de lentes). Hoy en día, se admite de manera general que, para dar respuesta a los problemas actuales -que han desbordado y traslapado las divisiones administrativas históricas-, la conducción de las políticas públicas se debe hacer de manera coordinada con una pluralidad de socios a diferentes niveles, tanto públicos como privados y asociativos (ONG). También se subraya el carácter policéntrico de la acción pública que la hace obligatoriamente pasar por estrategias comunicativas, participativas y deliberativas para su formación e implementación ${ }^{7}$. Nuestra definición contemporánea de la razón ha ido cambiando: ya no existe una sola razón "verdadera" sino que admitimos que pueden existir otras razones igualmente válidas, dependiendo el contexto y el lugar. En sumo se introdujo un cierto relativismo en nuestra cultura (occidental).

En términos prácticos, esto significa la creación de instancias y procedimientos de coordinación y deliberación tales como Comisiones, Mesa Redonda, Mesa de concertación, etc., con las cuales se pretende lograr la acción conjunta, trascendiendo el punto de vista particular, incluyendo el del Estado, para incluir otros actores en la formación e implementación de las acciones públicas, ya que la razón emerge del diálogo y resulta ser provisional y local. Y esto aparece como necesario ya que no solo estos actores pueden invocar intereses legítimos sino que disponen, a veces, de una verdadera capacidad de "chantaje" frente al Estado: disponen de la información, del conocimiento o de los re-

\footnotetext{
$6 \quad \mathrm{Al}$ respeto, sería interesante analizar las funciones ideológicas del uso de los términos "atraso" y "progreso" en la constitución del discurso político.

Papadopoulos Y., op. cit., p. 120
} 
cursos necesarios (o, en el caso colombiano, de la capacidad de coacción militar $^{8}$ ) para permitir llevar a cabo cualquier actividad en el dominio específico $^{9}$, o también impedírsela al Estado sin su consentimiento o colaboración. En otras palabras, la acción del Estado tiende hoy en día a diluirse y, a la vez, a necesitar del otro. El Estado no aparece como una instancia omnipotente y omnisciente separada y superior a una sociedad dominada por ésta. $\mathrm{Su}$ razón, que pretendía ser universal, ya no logra imponerse como evidente e indiscutible. Debemos pasar de una actividad basada en una razón mecánica, cuyo relojero era el Estado, a una razón compleja que se basa en la deliberación entre una pluralidad de actores involucrados y en donde el Estado es un actor más de la acción política ${ }^{10}$.

\section{Pasando por el Estado empresario}

¿Implica entonces esta dilución del Estado y pérdida de centralidad para la acción pública, el fin del Estado? Sí y no. Es efectivamente el caso, si consideramos el Estado desde la perspectiva moderna tradicional. En este sentido, es claro que este Estado debe desaparecer. La minimización del Estado fue una respuesta que representó, en la práctica, una primera tentación o intento. Fue la idea de considerar al Estado como un mero actor económico más, en competencia con otros, para la creación y distribución de bienes y de valores. Es el Estado empresario de los neoliberales. Este Estado empresario, como nueva metáfora, representa la respuesta a la crisis del Esta-

$8 \quad$ El éxito del chantaje y la penetración paramilitar (o guerrillera) en diversas instancias políticas y administrativas se debe en gran parte a la ausencia o falta de democratización y de transparencia de estas actividades. Por eso, la política estatal, el clientelismo tradicional y de los grupos armadas se dan la mano para defender y mantener sus intereses y posiciones de poder.

9 En el caso colombiano, es claro que la descentralización, con su inevitable fragmentación y división del poder que implica, ha aumentado la vulnerabilidad de las autoridades locales al chantaje de actores locales poderosos económica o militarmente. Unicamente con una democratización radical de las actividades públicas se logrará erradicar estas prácticas de corrupción.

10 Esta situación se traslada también en el campo académico del análisis de las políticas públicas. Autores (Roe, Hajer, Fischer) señalan la necesidad de utilizar una perspectiva analítica deliberativa o postempiricista, dejando atrás, la perspectiva -aun dominante en el área- neopositivista, en particular con la elección racional y la econometría en boga en el análisis de las políticas públicas. 
do burocrático-relojero a partir de los años 1970 y que recibió aun un mayor impulso con el derrumbe del Estado del capitalismo burocrático monopolista del antiguo bloque socialista, el cual había intentado imponer el paradigma relojero a todas las actividades sociales. El Estado empresario corresponde a la concepción de una sociedad organizada en torno a la creencia de la existencia de un principio de regulación natural: la mano invisible del mercado que equilibra las acciones individuales de los seres humanos. Es decir se pasa de la creencia en una razón objetiva, incorporada por el Estado moderno guiado por una ciencia positiva y que implementa sus soluciones mediante ingenieros sociales, a su abandono a una "ley natural": la creencia en un mercado autorregulado automático como mecanismo idóneo de la organización de la vida en sociedad y de la distribución justa de bienes y valores. Sin embargo, pasada la euforia de las privatizaciones, hoy, el desencanto con la mano invisible es también palpable.

Entonces ¿Qué hacer? Ante la imposibilidad de pensar en un nuevo relojero, es necesario repensar el papel y la concepción del Estado: si ni la planificación científica tradicional (razón positiva), ni la libertad de los mercados autorregulados (razón natural) son respuestas satisfactorias a la complejidad de la vida humana es preciso repensar el rol del Estado. Por eso la res- puesta a la pregunta inicial puede ser no: el Estado no tiene que desaparecer sino transformarse radicalmente. Es lo que nos proponemos explorar con la idea de la metáfora de un Estado como capitán del equipo de fútbol.

\section{Hacia el Estado futbolista}

En efecto, no se trata de que el Estado sea cualquier jugador, tampoco el árbitro, sino que éste sea el capitán del equipo. Con este cambio se trata de tomar en serio el paso del gobierno a la gobernanza, de la administración pública a la gestión pública y de la política pública a la acción pública. En esencia, se trata de pasar a modos de gobierno y de administración que tienen en cuenta la policentralidad o multipolaridad de los actores y de los saberes existentes en la sociedad y que deben ser partícipes deliberantes de la formación de la acción pública. Es la característica de la gobernanza: un proceso de formación, decisión e implementación de políticas públicas que involucra "organizaciones públicas no estatales, firmas comerciales, ciudadanos individuales y organizaciones internacionales" ${ }^{\text {"11 }}$. Es decir, es necesario reconocer la legitimidad de un pluralismo de actores más o menos autónomos los unos de los otros y que pueden y deben influir en la suerte de las acciones públicas. La necesaria cooperación entre la multiplicidad de los niveles de gobierno y la participación

11 Bresser Pereira L.C., "El modelo estructural de gobernanza pública”, en Reforma y Democracia, CLAD, №36, 2006, p.18 
de instancias privadas y no estatales para la construcción y prosecución efectiva de las metas públicas obliga a repensar seriamente el papel del Estado ${ }^{12}$. Aplicando la metáfora del futbolista, hay que construir un Estado-Zidane o un Estado-Ronaldo. Este Estado tiene que ser capaz de orientar y organizar el juego, anticipar las acciones del equipo adverso, y generar jugadas que, a la vez, son ganadoras solo si se construyen colectivamente. Es decir no tiene que ser un Estado-portero o de último recurso, o parte de un equipo en que todos los jugadores se la juegan en solitario a ver si por algún golpe del destino se salva (este sería más la idea del estado-empresario -todos contra todos- o del caudillismo - confiamos en uno solo jugador-).

De modo que podemos esbozar un paralelo entre las jugadas ganadoras con las acciones públicas efectivas que, además, garantizan gobernabilidad y legitimidad. Para un capitán de equipo de fútbol, cada problema que representa el equipo adverso es un problema nuevo, cambiante, con el cual las mismas jugadas no funcionan siempre. Tal como en el caso de los problemas sociales y políticos, sus movidas son cambiantes, innovadoras, logran aprender de las soluciones pasadas adaptándose a un nuevo contexto o provocando una situación inédita. Es necesario entonces renovar constantemente las jugadas, sorprender el otro con una jugada imprevisible o diferente. La capa- cidad del líder del juego se mide en el lograr aprovechar el potencial, la sabiduría y el conocimiento específico sobre el adversario directo de los demás compañeros para que colectivamente todos salgan ganadores mediante una solución que se construye sobre la marcha y lograra sorprender el adversario. Un partido de fútbol nunca es la repetición del anterior incluso cuando ningún jugador cambia. Es una microsociedad compleja. Del mismo modo, ninguna política pública es similar a otra.

Es así que en una sociedad, el Estado y la administración deben adaptarse a situaciones diversas, imprevistas, cambiantes. Deben olvidar la posibilidad de soluciones definitivas o demasiado previsibles. Por eso, la gobernanza implica asociar de manera participativa y deliberativa otros actores a la toma de decisión. En su nuevo rol, el Estado debe ser el ente que lidera la deliberación pública entre actores pertinentes. El Estado se encuentra frente a la tarea de gestionar (y no administrar) soluciones evolutivas en territorios problemáticos diversos, con contornos cambiantes, que no se dejan disecar ni horizontal (territorio políticoadministrativo) ni verticalmente (sector) de manera rígida y estable: se trata de pasar de las políticas de productos a las políticas de problemas. El territorio del problema de la economía no es el mismo que del medio ambiente, etc. Para esto, hay que imaginar las institucio-

$\overline{12}$ Se puede retomar aquí la idea de Niklas Luhmann de Estado como "primus inter pares". 
nes públicas (estatales) no como repertorios de soluciones sino como depósitos de medios que hay que usar de forma diferenciada según el problema. Por lo tanto se debe tener la flexibilidad para construir coaliciones "a geografía variable" con la participación de los ciudadanos. Pero, no se trata sencillamente de que el Estado siente alrededor de una mesa a los actores más activos o interesados en una temática. El Estado, cómo un capitán de equipo de fútbol, debe preocuparse también para que todos los jugadores estén en el partido. Por eso, el Estado debe fortalecer los grupos sociales que no son suficientemente representados para que participen en la acción. Con la deliberación, igualando el peso de los argumentos de cada actor, se permitiría así superar la predominación automática de los intereses más organizados. Ante la desigualdad de los actores, deben crearse escenarios deliberativos que equiparan el estatuto de los participantes, disminuyendo las asimetrías de poder entre ellos y que permite también evitar los riesgos de la dictadura de la mayoría.

La capacidad de asociar alrededor de la institución pública las partes comprometidas y afectadas, y ofrecerles, gestionarles, un marco para favorecer el descubrimiento de soluciones construidas mediante prácticas de deliberación y ajustes mutuos se vuelve enton- ces el elemento decisivo porque, de un lado, legitima la institución pública como actor clave de la política y, por el otro, le permite desarrollar efectivamente la acción pública ${ }^{13}$. De manera que la tarea del Estado, en sus diferentes niveles y componentes, es de gestionar la constitución de escenarios de democracia participativa y deliberativa para la formación e implementación de las acciones públicas, superando así el elitismo oligárquico de la democracia representativa.

América latina dispone en este campo de una serie de experiencias innovadores, con sus limitaciones, fracasos y éxitos, que solo se puede mencionar brevemente con algunos ejemplos. Las experiencias de democracia participativa en el Brasil, en particular con el conocido Presupuesto participativo, que permitió una renovación de los procesos de decisión en materia presupuestal. Las experiencias participativas en los municipios peruanos muestran interesantes procesos políticos que sustituyen el discurso de la acción directa y antiestatal de los movimientos sociales por el de la construcción de modalidades de colaboraciones abigarradas, ad hoc, entre instituciones públicas locales y sociedad civil ${ }^{14}$ renovando así el rol del Estado local. El autor muestra en particular la importancia de las autoridades muni-

13 Ver Duran P., Penser l'action publique, LGDJ, Paris, 1999, y Roth, A.-N. “ Formulación de políticas ", en IDCT, Formar para la democracia, IDCT-Alcaldía de Bogotá, Bogotá, 2003, pp. 21-26.

14 Ver Arroyo J., "La democracia municipal en la descentralización peruana 20012005: ¿exceso o insuficiencia de participación?”, en Reforma y Democracia, CLAD, 
cipales en su rol central de organizador y líder del proceso participativo. Igualmente, en el contexto diferente de la lucha contra la corrupción, hemos mostrado, como arreglos particulares entre diversos niveles político administrativos, organizaciones sociales y ciudadanos logran tener efectividad ${ }^{15}$.

Obviamente, estos procesos encuentran aún muchas dificultades y limitaciones. En particular, las experiencias y las reflexiones relativas al paso del nivel local al nivel regional o nacional están aun muy escasas. Igualmente, la deliberación, entendida como un proceso de debate argumentado entre participantes, está aún poco practicada. Sin embargo, este movimiento de renovación de los procesos político administrativos empieza reconfigurar la noción misma de democracia en América latina, e incluso empieza a exportarse en otros continentes: para citar un solo ejemplo de actualidad, la socialista Segolène Royal, designada candidata a la Presidencia de la República francesa a la elección de mayo 2007, sedujo a los militantes con sus intenciones de desarrollar modalidades de democracia participativa.

En conclusión, se considera que la acción pública contemporánea tiene que inscribirse en una práctica diversificada e inventiva, y en una reflexión teórica y política, que avanza hacia la constitución, a partir de las experiencias, de una teoría de la gestión pública democrática radical basada en el reconocimiento de la complejidad social y en la legitimación de formas institucionalizadas de deliberación pública. Esta teoría debe partir del reconocimiento del ocaso de la concepción autoritaria de un Estado omnisciente y omnipotente: el Estado no tiene las soluciones, pero puede disponer de los medios necesarios para facilitar la búsqueda colectiva de una solución que es necesaria concebir como provisional y evolutiva. La gestión pública debe transformarse en una labor de coordinación entre -y no de dirección sobre- actores pertenecientes a una sociedad considerada apta para la deliberación y la participación, y no como destinada a ser apenas representada por algunos de sus miembros. De la democracia representativa y la administración autoritaria, debemos avanzar hacia formas de democracia y gestión pública deliberativas: es decir a formas de autogestión o de autogobierno. De la monotonía mecánica de un reloj a la gracia y sutileza de una jugada maestra.

\footnotetext{
N³6, 2006, p.99-140. El Autor reporta la significativa anécdota de la Federación Campesino del Cusco que realizó un Congreso con el lema: "Qué podemos hacer sin el Estado?”, cuando se constató que no habría más prestamos agrarios por desaparición del Banco Agrario (p.132).

15 Mérat J., Roth A.-N., "La efectividad de las instituciones en la lucha contra la corrupción: la estrategia de los pactos de transparencia en Colombia", en Reforma y Democracia, CLAD, N³6, 2006, p.141-172.
} 